\title{
Multi-Frequency Encoding for Rapid Color Flow and Quadroplex Imaging
}

\author{
Oddershede, Niels; Gran, Fredrik; Jensen, Jørgen Arendt
}

Published in:

2007 IEEE Ultrasonics Symposium Proceedings

Link to article, DOI:

10.1109/ULTSYM.2007.279

Publication date:

2007

Document Version

Publisher's PDF, also known as Version of record

Link back to DTU Orbit

Citation (APA):

Oddershede, N., Gran, F., \& Jensen, J. A. (2007). Multi-Frequency Encoding for Rapid Color Flow and

Quadroplex Imaging. In 2007 IEEE Ultrasonics Symposium Proceedings (Vol. 1-6, pp. 1105-1108). IEEE. I E E

E International Ultrasonics Symposium. Proceedings https://doi.org/10.1109/ULTSYM.2007.279

\section{General rights}

Copyright and moral rights for the publications made accessible in the public portal are retained by the authors and/or other copyright owners and it is a condition of accessing publications that users recognise and abide by the legal requirements associated with these rights.

- Users may download and print one copy of any publication from the public portal for the purpose of private study or research.

- You may not further distribute the material or use it for any profit-making activity or commercial gain

- You may freely distribute the URL identifying the publication in the public portal 


\title{
Multi-frequency encoding for rapid color flow and quadroplex imaging
}

\author{
Niels Oddershede, Fredrik Gran, and Jørgen Arendt Jensen \\ Center for Fast Ultrasound Imaging, Ørsted•DTU, Build. 348, \\ Technical University of Denmark, DK-2800 Kgs. Lyngby, Denmark
}

\begin{abstract}
Ultrasonic color flow maps are made by estimating the velocities line by line over the region of interest. For each velocity estimate, multiple repetitions are needed. This sets a limit on the frame rate, which becomes increasingly severe when imaging deeper lying structures or when simultaneously acquiring spectrogram data for triplex imaging.

This paper proposes a method for decreasing the data acquisition time by simultaneously sampling multiple lines at different spatial positions for the color flow map using narrow band signals with disjoint spectral support. The signals are separated in the receiver by filters matched to the emitted waveforms and the autocorrelation estimator is applied. Alternatively, one spectral band can be used for creating a color flow map, while data for a number of spectrograms are acquired simultaneously. Using three disjoint spectral bands, this will result in a multi-frequency quadroplex imaging mode featuring a color flow map and two spectrograms at the same frame rate as a normal color flow map. The method is presented, various side-effects are considered, and the method is tested on data from a re-circulating flow phantom where a constant parabolic flow with a peak of 0.1 $\mathrm{m} / \mathrm{s}$ is generated with a flow angle of 60 degrees. A commercial linear array transducer is used and data are sampled using our RASMUS multi-channel sampling system. An in-vivo multifrequency quadroplex movie of the common carotid artery of a healthy male volunteer was created.

The flow phantom measurements gave a mean standard deviation across the flow profile of $3.1 \%, 2.5 \%$, and $2.1 \%$ of the peak velocity for bands at $5 \mathrm{MHz}, 7 \mathrm{MHz}$, and $9 \mathrm{MHz}$, respectively. The in-vivo multi-frequency quadroplex movie showed the color flow map, and the two independent spectrograms at different spatial positions. This enables studying the flow over an arterial stenosis by simultaneously acquiring spectrograms on both sides of the stenosis, while maintaining the color flow map. A frame rate of 21.4 frames per second was achieved in this in-vivo experiment.
\end{abstract}

\section{INTRODUCTION}

Since the introduction of the autocorrelation estimator for ultrasonic blood flow imaging by Kasai et. al. [1] in the mideighties, it has been possible to create full color flow maps (CFM) in real time. The method is robust and fairly simple to implement. The velocities are estimated line by line over the region of interest, and for each line, repeated transmissions are needed. The performance of the estimator is closely linked to the number of repetitions used to form the estimate [2]. An inherent trade-off between frame rate and performance therefore exist. When imaging deep structures like the heart, this might severely affect either the performance or the frame rate. Also, in the triplex imaging mode, where both CFM data and a spectrogram are shown, the time for data acquisition must be divided between multiple sets of data, increasing the significance of this problem even further.
The autocorrelation estimator essentially assumes a narrowband signal, and the variance of the velocity estimates decreases as the bandwidth is decreased [2]. Therefore a long sinusoidal narrow-band pulse is used for CFM. Nevertheless, ultrasound transducers are often designed fairly wide-band to ensure a good resolution in B-mode images. Using only a narrow frequency band for collecting CFM data does not exploit the available bandwidth efficiently.

This paper proposes a method for significantly decreasing the time spent on data collection for CFM by simultaneously sampling multiple lines using different frequency bands. The signals are then separated in the receiver by a simple filtering operation and the autocorrelation estimator is applied. The number of frequency bands $M$ depends on the available transducer bandwidth and the intensity limits set by the Food and Drug Administration [3]. The total time spent on CFM data acquisition will decrease by a factor of $M$, potentially increasing the frame rate with a factor of $M$. Alternatively, the proposed method can be used for simultaneously acquiring CFM data and spectrogram data for triplex imaging. This paper will demonstrate how data for an extension of triplex imaging, namely Multi-Frequency Quadroplex imaging (MFQ) featuring two independent spectrograms and a CFM, can be acquired during the time normally spent on acquiring a CFM.

Parallel receive beamforming has previously been proposed for decreasing the time spent on data acquisition for B-mode images [4] and for blood flow estimation [5]. Here a broadly focused transmit beam is emitted, and multiple receive beams are generated simultaneously by steering the beams in slightly different directions. While the parallel receive beamforming presented in [4] is limited to sample closely spaced lines within the transmitted beam, the proposed method provides a larger flexibility in where data is sampled, for instance enabling MFQ imaging. Ultimately, the two methods could be combined, introducing the possibility of making real-time CFMs of 3D volumes without ECG-gating.

\section{THEORY}

\section{A. Autocorrelation estimator}

The autocorrelation method for ultrasonic blood flow imaging was first introduced by Kasai et. al. [1]. Narrow-band pulses are repeatedly emitted along the same direction, and the received rf data are Hilbert transformed to give the in-phase and quadrature components. This results in a complex matrix $y(l, i)$, where $i$ is the transmission number and $l$ is the sample 
index along the rf line corresponding to depth $d_{l}=\frac{c}{2 f_{s}} l$. The axial velocity at a given depth can be estimated as [1]

$$
v_{z}=-\frac{c f_{p r f}}{4 \pi f_{0}} \arctan \left(\frac{\Im\{R(1)\}}{\Re\{R(1)\}}\right),
$$

where $c$ is the speed of sound, $f_{p r f}$ is the pulse repetition frequency, $f_{0}$ is the center frequency of the emitted narrowband signal, $\Re\{\cdot\}$ and $\Im\{\cdot\}$ denotes the real and imaginary parts, and $R(1)$ is the complex auto-correlation function of $y(l, i)$ at lag 1 , evaluated at a certain depth corresponding to $l=L_{d}$. This autocorrelation function can be estimated by

$$
\hat{R}(1)=\frac{1}{(N-1) N_{l}} \sum_{l=0}^{N_{l}-1} \sum_{i=0}^{N-2} y\left(l+L_{d}, i\right) y^{*}\left(l+L_{d}, i+1\right)
$$

which includes an averaging over $N_{l}$ rf samples. This has been shown to lower the variance of the estimated autocorrelation function, and thereby increase the accuracy of the velocity estimate [6].

By demanding that a whole period of the signal must be observed to distinguish the flow signal from that of a stationary structure, the minimum detectable velocity of an autocorrelation estimator is given by [2]

$$
v_{\text {min }}=\frac{c}{2} \frac{f_{p r f}}{N f_{0}} \text {. }
$$

This is a quite conservative demand, and $v_{\min }$ is not considered a rigid limit. According to the Nyquist sampling theorem, the maximum detectable velocity of an autocorrelation estimator is [2]

$$
v_{\max }=\frac{c}{2} \frac{f_{\text {prf }}}{2 f_{0}+B},
$$

where $B$ is the bandwidth of the emitted signal. The variance of the velocity estimate assuming a constant velocity, and under the assumption that no noise is present, can be approximated by [2]

$$
\sigma_{v}^{2}=\frac{c}{4 \pi^{2} f_{0}^{2}} \frac{f_{p r f}}{T}\left|v_{z}\right|
$$

where $T$ is the duration of the emitted pulse. Both the velocity range and the performance of the estimator are therefore dependent on the center frequency $f_{0}$.

\section{B. Spectral velocity estimation}

A spectrogram displays variation of the spectral content of the slow-time signal over time, hereby yielding a direct measure of the axial velocity of the moving blood. It can be estimated from the complex signal matrix $y(l, i)$ when the number of observations is sufficiently high. At a certain time instance $t=k / f_{p r f}$, the power spectrum over pulse repetitions is estimated from $N_{s}$ pulse repetitions and averaged over a number of rf samples $N_{l}$, which is known as the range gate. The segment size $N_{s}$ is chosen low enough to capture the frequency variations over time and high enough to give an acceptable spectral resolution. A window $w(i)$ is often applied
Emission 1 to $\mathrm{N}$

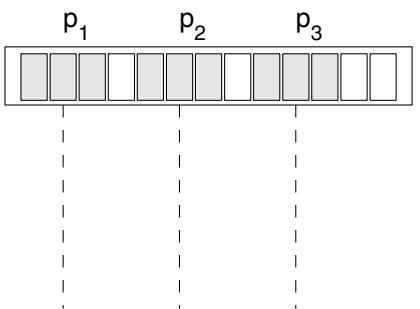

Fig. 1. The basic principle of the proposed method. Three different pulses $p_{1}(t), p_{2}(t)$, and $p_{3}(t)$ are emitted $N$ times and the received signals are separated in the receiver to simultaneously create three lines of the color flow map. The position of the transmitting sub-apertures are shifted and new lines are created.

to the data prior to the Fourier transform. The estimated power spectral density is given by

$$
\begin{aligned}
& \hat{P}_{y}\left(f_{p}, k\right)= \\
& \frac{1}{N_{l}} \frac{1}{N_{s}} \sum_{l=0}^{N_{l}-1}\left|\sum_{i=0}^{N_{s}-1} w(i) y\left(l+L_{d}, i+k\right) e^{-j 2 \pi f_{p} i / f_{p r f}}\right|^{2} .
\end{aligned}
$$

For a certain depth, corresponding to rf index $L_{d}, \hat{P}_{y}\left(f_{p}, k\right)$ is a function of frequency $f_{p}$ and time given by pulse repetition number $k$. Using a Hamming window given by

$$
w(i)=0.54-0.46 \cos \left(\frac{2 \pi i}{N-1}\right), \quad i=0,1, \ldots, N-1
$$

will lower the spectral side-lobes, coming from the limited observation time, to approximately $-40 \mathrm{~dB}$.

\section{Spatial encoding using frequency division}

The proposed method aims at increasing the frame rate of color flow mapping by simultaneously sampling data for multiple lines in the CFM. Fig. 1 (left) shows a linear array transducer simultaneously emitting $M=3$ different pulses $p_{1}(t), p_{2}(t)$, and $p_{3}(t)$ using different sub-apertures. Assuming the signals can be separated in the receiver, three different lines can be beamformed after each emission. The emission is repeated $N$ times at a given pulse repetition frequency $f_{p r f}$ and three lines in the CFM are created using the autocorrelation estimator [1]. The transmitting sub-apertures are slid across the aperture and the emissions are repeated until data for the entire CFM is collected. The time used for collecting data will in this example only be a third of that normally used.

Alternatively, the CFM data can be acquired as is usually done, using one narrow band signal $p_{1}(t)$. Simultaneously, data for two spectrograms can be acquired using the signals $p_{2}(t)$ and $p_{3}(t)$. These signals are repeatedly emitted from the same sub-apertures, continuously sampling along the same lines. Hereby a CFM and two spectrograms are acquired simultaneously yielding an expansion of triplex imaging named MultiFrequency Quadroplex imaging (MFQ). The data acquisition time will equal that normally spent on acquiring a CFM. At some point, a transducer element will be required to emit both 


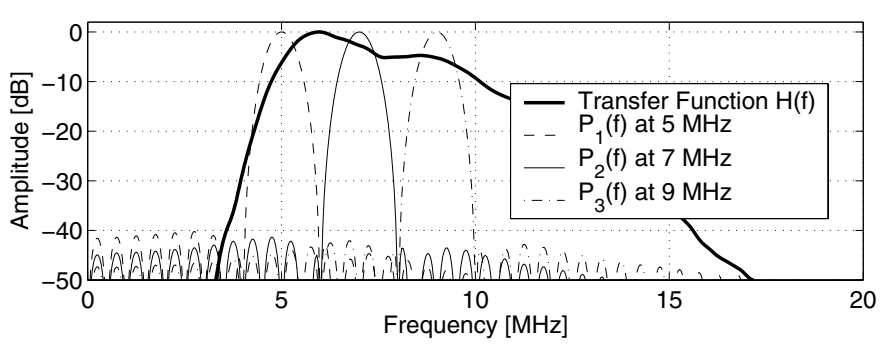

Fig. 2. The two-way system transfer function and the amplitude spectra of the three emitted pulses.

a delayed version of $p_{1}(t)$ and for instance $p_{2}(t)$. To use the entire amplitude range while emitting each signal, $p_{2}(t)$ and $p_{3}(t)$ are delayed so that they are emitted later than $p_{1}(t)$.

The emitted signals $p_{m}(t)$ are designed as simple narrowband pulses at different frequencies. They are given by

$$
p_{m}(t)=w(t) \sin \left(2 \pi f_{m} t\right), \quad 0<t<T
$$

for $m=[1,2,3]$, where $f_{m}$ is the center frequency of the $m$ th signal, and $T$ is the pulse duration. $w(t)$ is a window designed to reduce the spectral leakages into the other bands. A Hamming window, which has spectral side-lobes below -40 $\mathrm{dB}$, is used. The signals are then separated in the receiver by filters matched to the emitted signals, $h_{m}(t)=p_{m}(T-t), 0<$ $t<T$. The number of pulses $M$, which can be emitted, is determined by the transducer bandwidth compared to the bandwidth of the signals $p_{m}(t)$. Fig. 2 shows the transfer function of a commercial linear array transducer (thick line), and the amplitude spectra of three signals in (8) using $f_{m}=$ $[5,7,9] \mathrm{MHz}$ and $T=2 \mu$ s (thin lines). The spectral leakage is below $-40 \mathrm{~dB}$ for all bands.

The minimum detectable velocity is now limited by the lowest frequency band centered at $f_{1}$ through (3), and the maximum detectable velocity is limited by the highest frequency band $f_{M}$ through (4). The increased frame rate, thus, comes at the expense of a slightly decreased velocity range compared to a single narrow-band pulse. Furthermore, the method requires $M$ parallel beamformers, which is incorporated in most commercial high-end scanners at present, and a matched filtration of the channel RF-data.

\section{SimultaneOUS SAMPLING OF MULTIPLE CFM LINES}

The method was tested in a re-circulating flow rig, where a blood mimicking fluid was pumped at a constant velocity through a rubber tube submerged in a water tank. The physical setup of the phantom experiment is shown in Fig. 3, which also show the definition of the tube depth $z_{0}=33.5 \mathrm{~mm}$, the tube radius $R=6 \mathrm{~mm}$, and the beam-to-flow angle $\theta=60^{\circ}$. The peak velocity was $v_{0}=0.1 \mathrm{~m} / \mathrm{s}$.

The data acquisition was performed using a 128 element linear array transducer and the RASMUS multi-channel sampling system [7]. This system can emit arbitrary waveforms on 128 elements, and sample 64 transducer elements in realtime. To acquire data from all 128 elements, the emission is

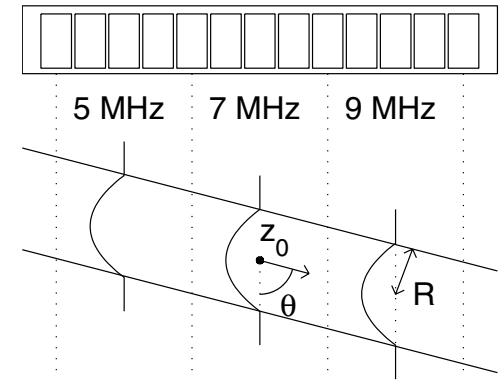

Fig. 3. Physical setup of the phantom experiment. The transducer is mounted at height $z=z_{0}$ over the tube center at an angle of $\theta=60^{\circ}$. The tube has radius $R$. The figure also shows the three regions of the CFM made at different frequency bands. Each region is sampled left to right and all regions are sampled simultaneously. The position of the three velocity profiles correspond to the profiles shown in Fig. 4.

repeated and multiplexing is used. This effectively halves the frame rate. The three signals shown in Fig. 2 were used and the parameters for the experiment are given in Table I.

TABLE I

PARAMETERS USED FOR THE PHANTOM EXPERIMENT.

\begin{tabular}{ll}
\hline Parameter & Value \\
\hline Transducer type & Linear array \\
Number of transducer elements & 128 \\
Transducer element pitch & $0.26 \mathrm{~mm}$ \\
Transducer element height & $4 \mathrm{~mm}$ \\
Elevation focus & $15 \mathrm{~mm}$ \\
Transfer function & See Fig. 2 \\
\hline Transmit focus depth & $28.7 \mathrm{~mm}$ \\
Number of transmit bands & 3 \\
Center frequencies, $f_{1}, f_{2}$, and $f_{3}$ & 5,7 , and 9 MHz \\
Pulse duration, $T$ & $2 \mu$ s \\
Amplitude tapering & Hamming window \\
Number of emit. elements / band & 22 \\
Transmit apodization & Tukey window \\
Number of receiving elements & $128(2 x 64$ through multiplexing) \\
Receive apodization & Hanning window (over 64 elem. \\
& cnt. around the current image line) \\
Sampling frequency, $f_{s}$ & $40 \mathrm{MHz}$ \\
Pulse repetition frequency, $f_{p r f}$ & $1.65 \mathrm{kHz}(3.3 \mathrm{kHz}$ incl. multiplex) \\
Number of shots per estimate, $N$ & $32(64$ incl. multiplexing) \\
Clutter filtering & Subtracting mean of $N$ signals \\
Number of CFM lines created & 33 \\
Inter-line spacing & $0.52 \mathrm{~mm}$ \\
\hline
\end{tabular}

The acquired data was processed as described in Section II. 17 repetitions of the entire sequence was processed, yielding 17 full color flow maps. The velocity profiles at the center line of each of the three regions (see Fig. 3) were extracted and the mean and standard deviation over the repetitions were found. These are shown in Fig. 4 along with the expected parabolic velocity profile.

Fig. 4 reveals a slightly higher standard deviation in the lower frequency bands. The standard deviations of the velocity estimates are averaged over the tube diameter yielding 3.1, 2.5 , and $2.2 \%$ of the theoretical peak velocity $v_{0}=0.1 \mathrm{~m} / \mathrm{s}$ for the $5 \mathrm{MHz}, 7 \mathrm{MHz}$, and $9 \mathrm{MHz}$ bands, respectively. The increase in standard deviation, although present, is not as large as predicted by (5). For this phantom experiment performed 

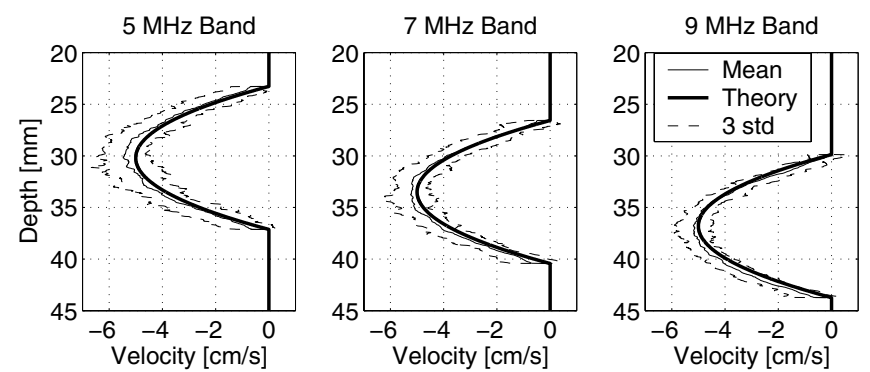

Fig. 4. Three resulting velocity profiles made at different frequency bands. The plot shows the expected velocity profile (thick line), the average of 17 profiles (thin line), and three times the standard deviation of the 17 profiles (dashed line).

in water a quite good signal-to-noise ratio is attained, and no frequency dependent attenuation is present. The increased standard deviation at lower frequency bands is therefore qualitatively in accordance with (5), which is derived assuming no noise.

\section{Multi-Frequency QuAdropleX IMAGING}

At present, triplex imaging is only possible at very low frame-rates due to the switching between acquisition of CFM data and spectrogram data. The proposed method can be used to solve this problem, and further to do MFQ imaging as described in Section II-C. The MFQ imaging was tested in vivo using a pulse repetition frequency of $f_{p r f}=12 \mathrm{kHz}(24$ $\mathrm{kHz}$ including multiplexing) and a transmit focus depth of 15 $\mathrm{mm}$. The remaining parameters were set as described in Table I. An examples from the common carotid artery of a healthy male volunteer is shown in Fig. 5. The CFM is made using the $5 \mathrm{MHz}$ band and is composed of 33 lines, each sampled 16 times (32 including multiplexing), and the B-mode image is made from 65 emissions. The leftmost spectrogram was made using the $7 \mathrm{MHz}$ band and the rightmost using the 9 $\mathrm{MHz}$ band. Each spectrogram estimate was made from 64 lines using the method described in II-B. The frame rate was 21.4 $\mathrm{Hz}$. While the B-mode data was acquired (which happens 21.4 times each second), no spectrogram data are available. This is a result of the B-mode emissions using the entire transducer bandwidth. In Fig. 5, this is seen as gaps in the spectrogram. These gaps are not inherent for the proposed method, but are dependent on how the emissions are ordered, and there are multiple ways of avoiding these gaps [8], [2], [9].

\section{Conclusion}

A data acquisition method for simultaneously sampling multiple lines using different narrow frequency bands has been presented. The method can be used for increasing the frame rate of CFMs or for MFQ imaging, featuring a CFM and two independent spectrograms. The MFQ imaging mode yields the possibility of studying the change in flow over an arterial stenosis by placing a range gate at both sides of the stenosis and monitoring the flow over time, while still maintaining the CFM.



Fig. 5. Multi-Frequency Quadroplex image of the common carotid artery during systole. The upper image shows the color flow map using the $5 \mathrm{MHz}$ band, the left spectral Doppler shows the $7 \mathrm{MHz}$ band using the leftmost range gate, and the right spectral Doppler shows the $9 \mathrm{MHz}$ band using the rightmost range gate.

\section{ACKNOWLEDGMENTS}

This work was supported by grant 26-04-0024 from the Danish Science Foundation, by the Danish research council for Technology and Production grant 274-05-0327, and by BK Medical, Herlev, Denmark. MD Kristoffer Lindskov Hansen is thanked for performing the in-vivo data acquisition.

\section{REFERENCES}

[1] C. Kasai, K. Namekawa, A. Koyano, and R. Omoto. Real-time twodimensional blood flow imaging using an autocorrelation technique. IEEE Trans. Son. Ultrason., 32:458-463, 1985.

[2] J. A. Jensen. Estimation of Blood Velocities Using Ultrasound: A Signal Processing Approach. Cambridge University Press, New York, 1996.

[3] FDA. Information for manufacturers seeking marketing clearance of diagnostic ultrasound systems and transducers. Technical report, Center for Devices and Radiological Health, United States Food and Drug Administration, 1997.

[4] D. P. Shattuck, M. D. Weinshenker, S. W. Smith, and O. T. von Ramm. Explososcan: A parallel processing technique for high speed ultrasound imaging with linear phased arrays. J. Acoust. Soc. Am., 75:1273-1282, 1984.

[5] J. W. Allison. Parallel Doppler processing in ultrasonic flow imaging. PhD thesis, Duke University, Durham, NC, 1987.

[6] T. Loupas, J. T. Powers, and R. W. Gill. An axial velocity estimator for ultrasound blood flow imaging, based on a full evaluation of the Doppler equation by means of a two-dimensional autocorrelation approach. IEEE Trans. Ultrason., Ferroelec., Freq. Contr., 42:672-688, 1995.

[7] J. A. Jensen, O. Holm, L. J. Jensen, H. Bendsen, S. I. Nikolov, B. G. Tomov, P. Munk, M. Hansen, K. Salomonsen, J. Hansen, K. Gormsen, H. M. Pedersen, and K. L. Gammelmark. Ultrasound research scanner for real-time synthetic aperture image acquisition. IEEE Trans. Ultrason., Ferroelec., Freq. Contr., 52 (5):881-891, May 2005.

[8] K. Kristoffersen and B. A. J. Angelsen. A time-shared ultrasound Doppler measurement and 2-D imaging system. IEEE Trans. Biomed. Eng., BME35:285-295, 1988.

[9] J. A. Jensen. Spectral velocity estimation in ultrasound using sparse data sets. J. Acoust. Soc. Am., vol 120(1):211-220, 2006. 